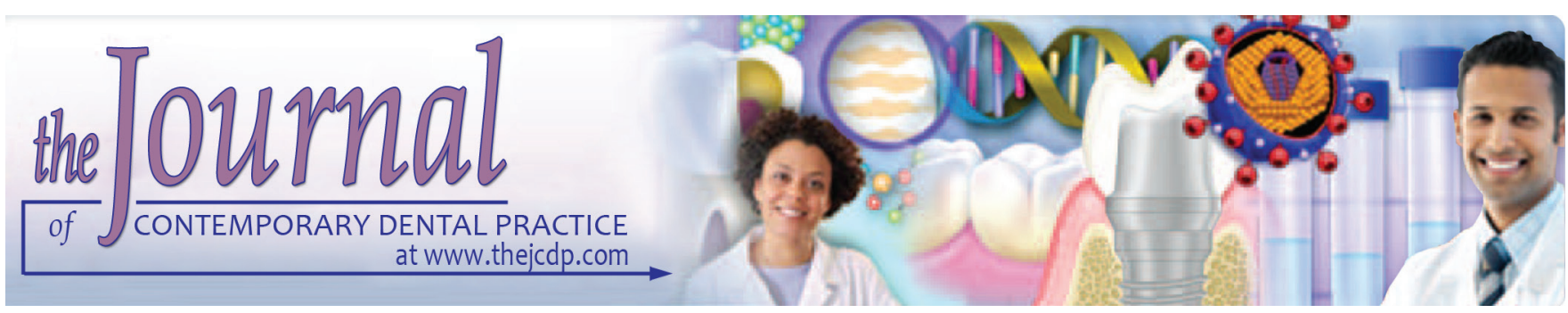

\title{
Evaluation of Effect of Connector Designs in Implant Tooth-supported Fixed Partial Denture: A Two-dimensional Finite Element Analysis
}

${ }^{1}$ Srinivasa Gowda, ${ }^{2}$ Dilip D Quadras, ${ }^{3}$ Rajeet S Sesappa, ${ }^{4}$ Vidyachandra Katapadi, ${ }^{5}$ Lalit Kumar, ${ }^{6}$ Dinraj Kulkarni, ${ }^{7}$ Nitu Mishra

\begin{abstract}
Aim: The aim of this study was to evaluate the effect of connector designs on scale and distribution pattern of the stress generated in the supporting bone of implant tooth-supported three-unit fixed partial denture in distal extension situation.
\end{abstract}

Materials and methods: Three-unit fixed partial denture geometric models with implant abutment in second molar, pontic in first molar, and second premolar as mesial abutment in distal extension situations were evaluated using a two-dimensional (2D) finite element analysis. Three models were designed and constructed with mesial and distal rigid connectors, mesial nonrigid connector, and distal nonrigid connector respectively, using the software ANSYS, version 10.0 (University Intermediate). The models were analyzed to determine the maximum equivalent von Mises stress at five critical zones (maximum value) under static axial loading $(240 \mathrm{~N})$ after meshing and assigning the material properties.

Results: The maximum stress concentration values at mesial and distal alveolar crest of the implant-supporting bone were

\footnotetext{
${ }^{1}$ Department of Prosthodontics, Armed Forces Medical College Maharashtra University of Health Sciences, Pune, Maharashtra India

${ }^{2}$ Department of Orthodontics and Dentofacial Orthopedics Srinivas Institute of Dental Sciences, Mangaluru, Karnataka, India

${ }^{3,4}$ Department of Oral and Maxillofacial Surgery, Srinivas Institute of Dental Sciences, Mangaluru, Karnataka, India

${ }^{5}$ Department of Prosthodontics, Dr. Harvansh Singh Judge Institute of Dental Sciences \& Hospital, Panjab University Chandigarh, India

${ }^{6}$ Department of Oral and Maxillofacial Pathology, M. A. Rangoonwala College of Dental Sciences \& Research Centre Pune, Maharashtra, India

${ }^{7}$ Department of Oral and Maxillofacial Pathology, Modern Dental College \& Research Centre, Indore, Madhya Pradesh, India
}

Corresponding Author: Srinivasa Gowda, Department of Prosthodontics, Armed Forces Medical College, Maharashtra University of Health Sciences, Pune, Maharashtra, India, Phone: +919049866969, e-mail: pfasrinivasa@gmail.com
60.59 and 68.57 MPa, respectively, in Model No 1. The high equivalent von Mises stress concentration values at the mesial and distal alveolar crest of the implant-supporting bone were 1.65 and $0.747 \mathrm{MPa}$ with $0.1 \mathrm{~mm}$ vertical movement and 7.88 and $9.34 \mathrm{MPa}$ with $0.5 \mathrm{~mm}$ vertical movement of the connector respectively, in Model No 2. The high equivalent von Mises stress concentration values at mesial and distal alveolar crest of the implant-supporting bone were 10.45 and $3.43 \mathrm{MPa}$ with $0.1 \mathrm{~mm}$ vertical movement and 4.50 and $5.71 \mathrm{MPa}$ with $0.5 \mathrm{~mm}$ vertical movement of the connector respectively, in Model No 3.

Conclusion: In the supporting bone around the implant in Model No 1, the maximum von Mises stress concentrations were displayed in the crestal zones. In the supporting bone around the implant abutment, the von Mises stress concentrations were minimal toward the apical third zone in all the models. The stress concentrations were minimal in the supporting bone around the implant and the natural tooth in the models with nonrigid connector.

Clinical significance: When implant is used as distal abutment in three-unit implant tooth-supported fixed partial denture with pontic at first molar in distal extension situation, it is recommended to place the nonrigid connector in the mesial side of the distal implant abutment.

Keywords: Connector, Geometric model, von Mises stress.

How to cite this article: Gowda S, Quadras DD, Sesappa RS, Katapadi V, Kumar L, Kulkarni D, Mishra N. Evaluation of Effect of Connector Designs in Implant Tooth-supported Fixed Partial Denture: A Two-dimensional Finite Element Analysis. J Contemp Dent Pract 2018;19(6):669-674.

Source of support: Nil

Conflict of interest: None

\section{INTRODUCTION}

Rehabilitation of distal extension situations in mandible is a complex procedure due to various anatomical and biomechanical factors like lack of adequate support, retention, and stability for the resultant prosthesis. Such situation may be managed with removable prosthesis, fully implant-supported prosthesis, natural tooth, and 
implant-supported fixed prosthesis. Among these, natural tooth and implant-supported fixed prosthesis are a viable option as described by various authors. ${ }^{1,2}$ The advantages of combining natural tooth and implant abutments are effective rehabilitation with minimum number of implants, thus minimizing surgical trauma and also providing a cost-effective prosthetic treatment option.

The problem arises with the force distribution and degree of movement with implant fixture and natural tooth due to the presence of periodontium in natural tooth. ${ }^{1-3}$ A well-supported natural tooth has a mobility of 50 to $200 \mu \mathrm{m}$ primarily due to the periodontal ligament. An osseointegrated implant may move only up to $10 \mu \mathrm{m}$ primarily due to bone flexibility. Due to the differential mobility, when teeth and implant abutments are joined in fixed partial denture, "the implant supports the tooth."4-6 It has been advocated that anatomical movements of tooth will create a bending momentum through the implant into the bone because the fixed partial denture joining them acts as a cantilever there. ${ }^{3-5}$ Many implant dentists feel that lesser the flexibility of the connector in the denture, higher the support between the natural tooth and implant. Because of close proximity at the bone implant junction, the load applied to the implant is directly transmitted to the supporting bone. Therefore, the biologic reaction of the osseous tissue is linked with implant longevity. This situation will lead to drawbacks like implant overloading, enhanced bone resorption, loss of osseointegration, and failure of fixed prosthesis and implant components. 5,6

This makes it necessary to break the stress generated around the implant by using a nonrigid connector. Thus, the design of the connector has significant influence on the stress distribution. ${ }^{4,5}$ However, there is inadequate literature about the use of nonrigid connectors in tooth implant-supported fixed prosthesis. Melo et $\mathrm{al}^{6}$ stated that the use of flexible connector in tooth and implantsupported fixed prosthesis does not reduce stress / strain in the implant-supporting bone. Menicucci et $\mathrm{al}^{7}$ stated that the periodontium has an important function in load distribution of implant. On the contrary, the role of the nonrigid connectors (especially the location of nonrigid connector) between the natural tooth and implant has not been verified in detail. With this background in mind, it was planned to evaluate the effect of connector designs on the distribution pattern and magnitude of stress under axial loading in the tooth and implantsupported fixed prosthesis using a 2D finite element method (FEM).

\section{MATERIALS AND METHODS}

Fixed partial denture with second premolar tooth as mesial abutment, pontic at missing first molar, and distal implant abutment at missing second molar in lower arch distal extension situation was analyzed. The geometric models were created by the software ANSYS: Version 10.0 (The University of Queensland and QCIF, School of Engineering, JKMRC and DVC Research, Australia) and analyzed by the 2D finite element analysis (the CAD-CAM Department, MAEER's Maharashtra Institute of Technology, Pune, India).

The procedure involved modeling of surface on the component from surface edges and curves to make a 2D model of the object followed by generation of nodal coordinates ( $\mathrm{X}$ and $\mathrm{Y}$ ) to the elements. This was followed by assigning the material properties (Poisson's ratio and modulus of elasticity) to enable the software to solve the equation and application of boundary conditions to limit translation or rotation in an appropriate manner (Table 1). Then application of loading was done with satisfactory accuracy, so that the geometry is converted to finite element form which was solved by software. The data generated during the solving stage were converted to a form that is easily understood by the operator. The stress generated was visualized on colored contour maps. The deformed shapes were visualized and stress was calculated for each node by von Mises criteria.

Wheeler's ${ }^{8}$ measurements were followed for constructing both premolar teeth. Mandibular molar region height was determined as $23 \mathrm{~mm}$, bone cortex thickness was $1.5 \mathrm{~mm}$, and periodontal membrane width was $0.2 \mathrm{~mm}$. The axes of natural teeth and implant $(13 \mathrm{~mm}$ $\times 3.75 \mathrm{~mm}$, Nobel Replace Select, Nobel Biocare, Zurich, Switzerland) in models were prepared in reference to compensatory curves (Fig. 1). The preparation of natural teeth and creation of metal ceramic restorations were done as per prosthetic guidelines. ${ }^{9}$ The $0.5 \mathrm{~mm}$ thickness nickel-chromium alloy core was veneered with $1.5 \mathrm{~mm}$ thickness porcelain. The nonrigid connector was placed with deep preparation. Slide-type attachment (T-123, Metalor, Neuchatel, Switzerland) was the nonrigid connector. The nonrigid connector was $2 \mathrm{~mm}$ length in vertical plane for all the models.

Table 1: Material properties assigned

\begin{tabular}{lll}
\hline Material & $\begin{array}{l}\text { Young's modulus } \\
(\mathrm{E})(\mathrm{GPa})\end{array}$ & $\begin{array}{l}\text { Poisson's } \\
\text { ratio }(\mathrm{V})\end{array}$ \\
\hline Enamel & 84 & 0.33 \\
Dentin & 18.6 & 0.31 \\
Pulp & 0.002 & 0.45 \\
Periodontal ligament & 2 & 0.45 \\
Cancellous bone & 1.5 & 0.30 \\
Cortical bone & 15 & 0.30 \\
Titanium & 110 & 0.33 \\
Ni-Cr alloy & 218 & 0.33 \\
Porcelain & 69.0 & 0.28 \\
\hline
\end{tabular}




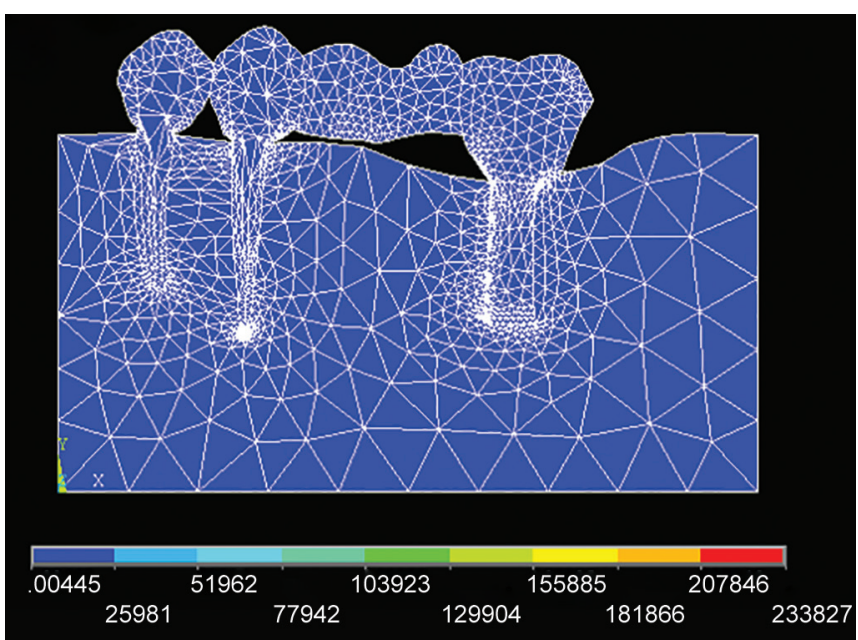

Fig. 1: Model No 1: Mesial and distal rigid connectors

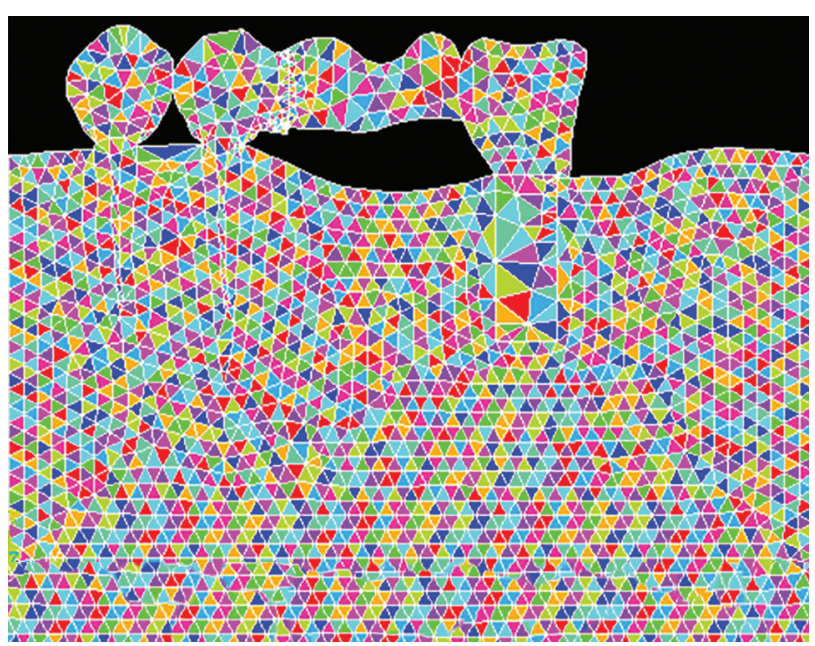

Fig. 3: Model No 3: Mesial rigid connector and distal nonrigid connector

In Model No 1, both the abutments were connected rigidly at both connectors (Fig. 1). In Model No 2, the nonrigid connector was placed in the distal side of the mesial abutment (nonrigid mesial connector) and distally rigid connector (Fig. 2). In Model No 3, the nonrigid connector was placed in the mesial side of the implant abutment (distal nonrigid connector) and mesial rigid connector (Fig. 3). The nonrigid connectors were having vertical direction movements of 0.1 and $0.5 \mathrm{~mm}$. The element type used was Plane 42 with degrees of freedom, translations in $\mathrm{x}$ and $\mathrm{y}$ directions. Care was taken to concentrate the mesh pattern in the region which was to be studied (i.e., in the supporting bone). The models were converted to 2D mathematical models. All the structures depicted in the model, such as cancellous bone, compact bone, the teeth, and the implant were linearly elastic and consistent. However, cortical bone contains anisotropic material characteristic and regional stiffness variation. Sufficient data are unavailable to establish the principle axis of anisotropy and so it is assumed to be isotropic.

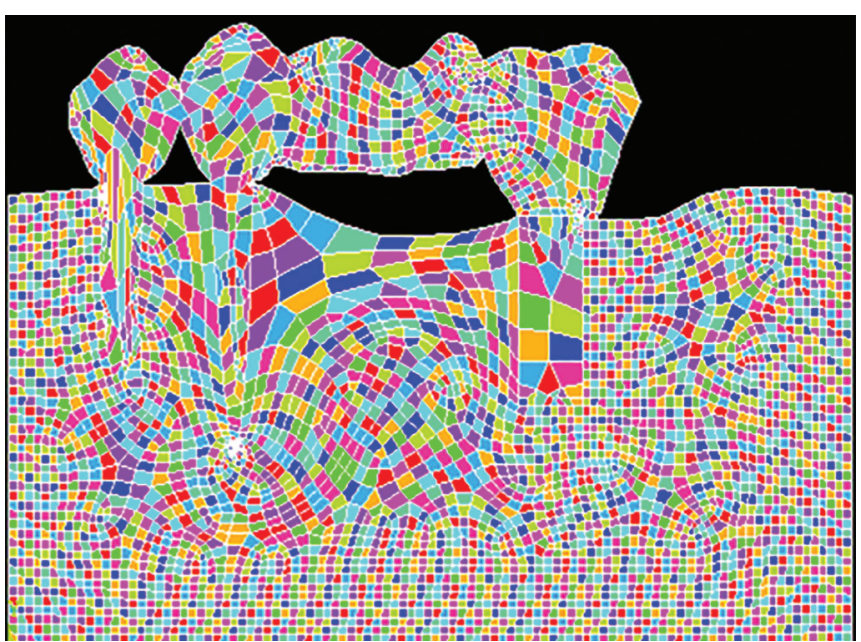

Fig. 2: Model No 2: Mesial nonrigid connector and distal rigid connector

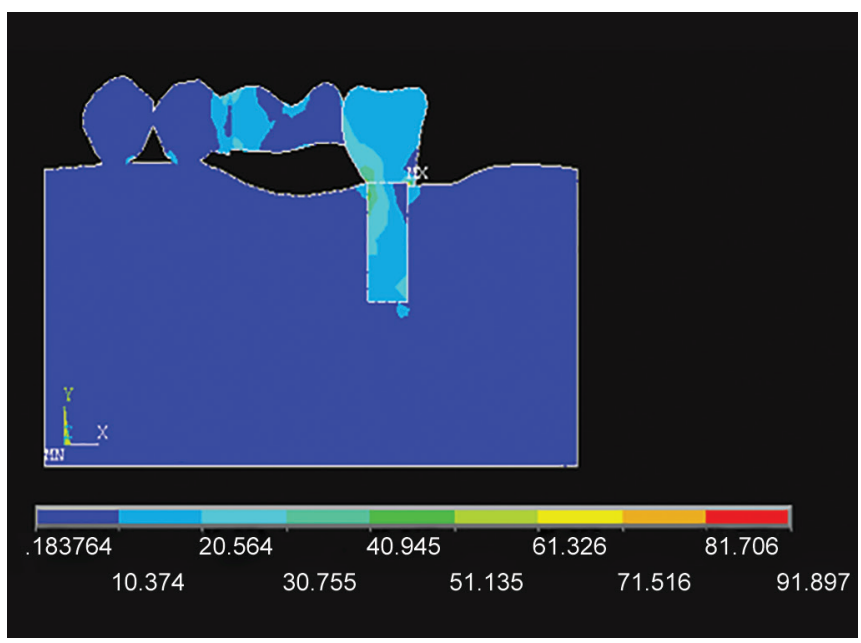

Fig. 4: The stress distribution and concentrations in Model No 1

In vertical plane, a nondynamic linear analysis was performed on 2D models with a masticatory force of $240 \mathrm{~N}$. The models were analyzed to determine the von Mises stress, which summarizes the tensile and shear stress generated in the implant-supporting bone for each model at five critical zones (maximum value) under static vertical loading.

\section{RESULTS}

Model No 1 (mesial and distal rigid connectors): Peak stress concentrations were displayed in mesial and distal crestal portion of the implant-supporting bone. The von Mises stress concentration values at mesial and distal bone crest of the implant-supporting bone were 60.59 and 68.57 MPa respectively. The von Mises stress concentration values at mesial and distal crestal area of the supporting bone of natural tooth were 20.839 and 17.42 MPa (Fig. 4).

Model No 2 (mesial nonrigid connector and distal rigid connector): The von Mises stress concentration values at mesial and distal crestal area of implant-supporting bone 


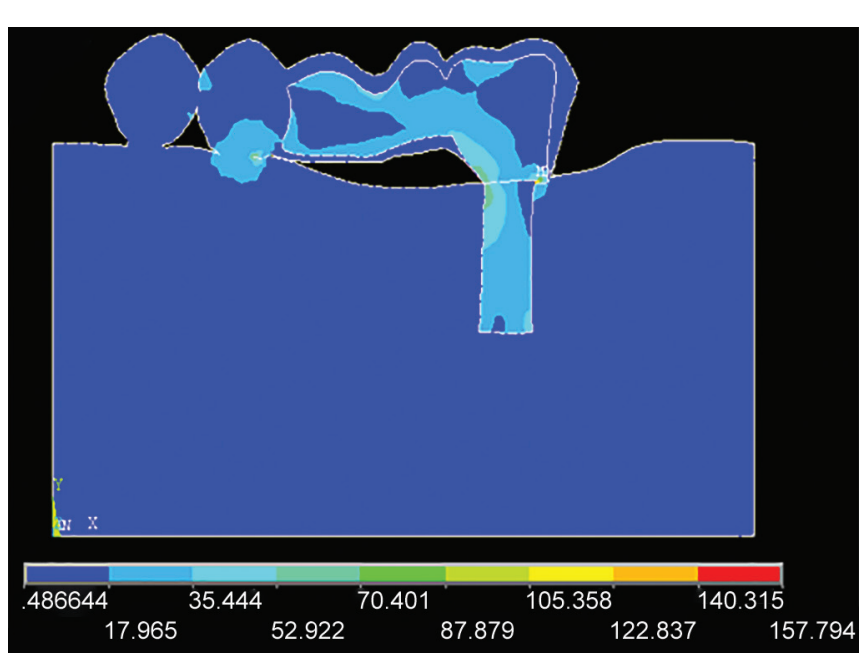

Fig. 5: The stress distributions and concentrations in Model No 2

were 1.65 and $0.747 \mathrm{MPa}$ with $0.1 \mathrm{~mm}$ vertical movement of the connector and 7.88 and $9.34 \mathrm{MPa}$ with $0.5 \mathrm{~mm}$ vertical movement of the connector. The von Mises stress concentration values at proximal crestal area of the supporting bone of natural tooth were 4.51 and $3.80 \mathrm{MPa}$ with $0.1 \mathrm{~mm}$ vertical movement of the connector and 2.2 and $2.7 \mathrm{MPa}$ respectively with $0.5 \mathrm{~mm}$ vertical movement of the connector (Fig. 5).

Model No 3 (distal nonrigid connector and mesial rigid connector): The high equivalent von Mises stress concentrations at the mesial and distal crestal area of the implant-supporting bone were 10.45 and $3.43 \mathrm{MPa}$ with $0.1 \mathrm{~mm}$ vertical movement and 4.50 and $5.71 \mathrm{MPa}$ with $0.5 \mathrm{~mm}$ vertical movement of the connector. The von Mises stress concentration values at proximal crestal areas of the supporting bone of natural tooth were 2.75 and 1.93 $\mathrm{MPa}$ with $0.1 \mathrm{~mm}$ vertical movement of the connector and 13.67 and $13.58 \mathrm{MPa}$ with $0.5 \mathrm{~mm}$ vertical movement of the connector (Fig. 6).

\section{DISCUSSION}

From an engineering point of view, tooth implantsupported fixed prostheses should be considered as a multicomponent structure consisting of a complex geometry. ${ }^{10,11}$ Whenever such a complex geometry is acted upon by a system of forces, it produces a variety of reactions. In an implant-supported situation, these reactions can be either stimulatory loading or pathologic overload, depending on the magnitude of loads acting on the implant. This should be considered during the planning and selection of tooth implant-supported fixed prostheses in implant dentistry. ${ }^{1-3}$ Stress analysis in implants becomes critical as the bone is less equipped to bear stress with an absence of the cushioning effect of the periodontal ligament. ${ }^{12,13}$ From critical assessment, the longevity of an implant is the manner in which the

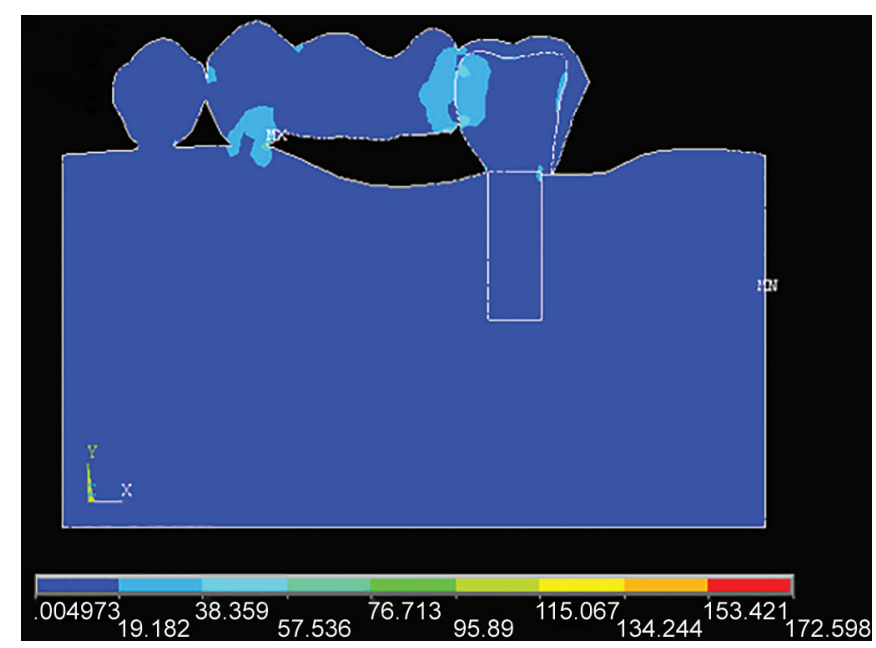

Fig. 6: The stress distributions and concentrations in Model No 3

mechanical stress is transferred from prosthesis to bone through implant. It must be noted that implant cannot be stressed beyond fatigue fracture. The design of the connectors in tooth implant-supported fixed prostheses is one among the factors which influence the magnitude and the distribution pattern of stress in bone. ${ }^{12,13}$ In view of this, it is necessary to biomechanically assess and validate the tooth implant-supported fixed partial denture connector designs which will be most conducive to its performance with respect to the bone under the varying loading conditions.

There are several methods like FEM, photo elastic stress analysis method, etc., available to evaluate the magnitude and distribution pattern of stress in the supporting bone around the abutments. ${ }^{12,13}$ Among these, finite element analysis is preferable, as it accurately simulates the real-life situation which can be studied in short time duration..$^{14}$ Finite element analysis has been established as a very useful tool in understanding and evaluating the stress in a system like bone-implant interface. ${ }^{14,15}$ Usually for ductile materials, von Mises failure theory is adopted, as it gives a realistic value of stress and strains corresponding to the actual material behavior. ${ }^{13}$ A computer imitation is studied with several simplifications related to physical and mechanical properties of bone, implant, and tooth. Advantages of finite element analysis are that it is a nondestructive method of testing which employs simulation of conditions with greater accuracy. Physical and mechanical properties in adjacent elements may not be similar. This allows the method to be applied to bodies composed of several materials. Irregularly formed margins may be analyzed using straight side elements or coordinated curved margin elements. There is variation in the size of the elements. This helps in the expansion of element grid as per requirement. Thus, discontinuous surface loading boundary conditions can be easily managed. ${ }^{13,14}$ 
Table 2: von Mises stress at crestal zones of supporting bone (in MPa)

\begin{tabular}{|c|c|c|c|c|c|}
\hline & & \multicolumn{2}{|c|}{ Implant } & \multicolumn{2}{|c|}{ Natural tooth } \\
\hline & & Mesial & Distal & Mesial & Distal \\
\hline Model 01 (mesial and distal rigid connectors) & & 60.59 & 68.57 & 20.839 & 17.42 \\
\hline \multirow[t]{2}{*}{ Model 02 (mesial nonrigid connector) } & $0.1 \mathrm{~mm}$ vertical movement & 1.65 & 0.747 & 04.51 & 03.80 \\
\hline & $0.5 \mathrm{~mm}$ vertical movement & 07.88 & 09.34 & 2.2 & 2.7 \\
\hline \multirow[t]{2}{*}{ Model 03 (distal nonrigid connector) } & $0.1 \mathrm{~mm}$ vertical movement & 10.450 & 3.431 & 2.75 & 1.93 \\
\hline & $0.5 \mathrm{~mm}$ vertical movement & 04.50 & 05.71 & 13.67 & 13.58 \\
\hline
\end{tabular}

In the current study, it was seen that the maximum stress concentrations were in the crestal zones and cervical zones of the supporting bone around the implant abutment in all the models (Table 2). The implant movements in supporting bone are lesser than millimeter level due to the osseointegration of implant with bone. ${ }^{16,17}$ When masticatory forces intrude the natural tooth into alveolus, they may cause stress within the implant-supporting bone. The implant's rotation center is more occlusal at the crestal bone level in comparison with tooth. Since the implant moves around this cervical rotation center, stress amassing occurs in the crestal zone of supporting bone. ${ }^{16,17}$ And also, there is formation of the supporting bone by outer cortical and inner cancellous bone, with different modulus of elasticity which adds up to the stress concentration in the supporting bone. ${ }^{14,15}$ As we go apically, the stress concentrations were reduced and minimal to no stress was found in the apex of the implant. This supported the findings of Bechelli ${ }^{16}$ who advocated that the apical portion of implant fixture is in contact with minimum cortical bone when compared with the cervical portion of implant fixture. This can be attributed to the fact that the difference in values of Young's modulus of different materials leads to a difference in the distribution pattern of stress among the materials. Titanium (implant material) showed the maximum stress followed by cortical bone and the minimal stress was seen in the cancellous bone.

Comparatively greater stress concentration values were observed in the model with the rigid connectors than the models with nonrigid connector (Figs 4 to 6). These results confirm the studies of Bechelli ${ }^{16}$ and Ozcelik and Ersoy. ${ }^{17}$ It was noted that providing optimum vertical movement in the connector allows wider stress dissipation to occur, relieving the supporting bone around the implant from the undue stress and strain. Bechelli ${ }^{16}$ advocated that the nonrigid connector may be placed on the mesial aspect of the implant abutment in the tooth implant-supported fixed prosthesis, avoiding the torque effects on implant. He further advocated that it allows natural movements of tooth and it will not affect the implant, if the tooth fails due to complicated periodontitis. These findings were present in our study in which a decreasing pattern of the stress concentration in the implant-supporting bone was seen in Model No 3 (Fig. 3). ${ }^{16-18}$

Limitations of this study were that the supporting tissues were assumed to be consistent, uniform, and linearly elastic structures, assumption of the perfect osseointegration of implant with bone, and the fabrication of a suitable model is very tough due to the intrinsic complexity of the host. Hence, quantitative data of the study may not be applied directly to clinical practice. The masticatory forces were loaded in vertical plane in this static study, but the masticatory forces are dynamic and oblique in nature with respect to occlusal plane. So a long-term in vivo study to support the above tests must be carried out.

\section{CONCLUSION}

Within the restrictions of this study, the following conclusions were drawn:

- Model with rigid connectors displayed maximum resultant stress concentrations in the crestal zone of the supporting bone around the implant abutment.

- The models with nonrigid connector displayed minimal resultant stress concentrations in the crestal zone of the supporting bone around the implant abutment.

- The amounts of vertical movements simulated, either 0.1 or $0.5 \mathrm{~mm}$, do not show much difference on the concentrations of stress in the implant-supporting bone.

- Various connector designs and locations minimally affect the stress concentrations in the supporting bone around the second premolar.

\section{CLINICAL SIGNIFICANCE}

It may be recommended that when the mesial natural tooth and distal implant are used together as abutments for pontic in three-unit fixed partial denture in distal extension situation, the flexible connector may be placed on the distal aspect of the pontic.

\section{REFERENCES}

1. Misch, CE. Dental implant prosthetics. 3rd ed. St. Louis (MO): Elsevier Mosby; 2005. 
2. Weinberg LA, Kruger B. Biomechanical considerations when combining tooth-supported and implant-supported prostheses. Oral Surg Oral Med Oral Pathol 1994 Jul;78(1):22-27.

3. Lundgren D, Laurell L. Biomechanical aspects of fixed bridgework supported by natural teeth and endosseous implants. Periodontol 20001994 Feb;4:23-40.

4. Rangert B, Jemt T, Jorneus J. Forces and moments on Branemark implants. Int J Oral Maxillofac Implants 1989 Fall;4(3):241-247.

5. Skalak R. Osseointegration biomechanics. J Oral Implantol 1986;12(3):350-356.

6. Melo C, Matsushita Y, Koyano K, Hirowatari H, Suetsugu T. Comparative stress analyses of fixed free-end osseointegrated prostheses using the finite element method. J Oral Implantol 1995;21(4):290-294.

7. Menicucci G, Mossolov A, Mozzati M, Lorenzetti M, Preti G. Tooth-implant connection: some biomechanical aspects based on finite element analyses. Clin Oral Implants Res 2002 Jun;13(3):334-341.

8. Wheeler, RC. Dental anatomy, physiology, and occlusion. 7th ed. Philadelphia (PA): Saunders; 1974.

9. Naylor, WP. Introduction to metal ceramic technology. Chicago (IL): Quintessence; 1992.

10. Shilinburg,HT.; Brackett, S.; Whitsett, L.;Sumiya,H.; Brackett, S. Fundamentals of fixed prosthodontics. 3rd ed. Chicago (IL): Quintessence; 1997.
11. Chandrupatala, TR.; Belegundu, AD. Introduction to finite elements in engineering. 2nd ed. Upper Saddle River (NJ): Prentice Hall; 1997.

12. Adams, V.; Askenazi, A. Building better products with finite element analysis. Santa Fe: On word press; 1999.

13. DeTolla DH, Andrena S, Patra A, Buhite R, Comella B. Role of the finite element model in dental implants. J Oral Implantol 2000;26(2):77-81.

14. Misch CM, Ismail YH. Finite element stress analysis of toothto-implant fixed partial denture designs. J Prosthodont 1993 Jun;2(2):83-92.

15. Breeding LC, Dixon DL, Sadler JP, McKay ML. Mechanical considerations for the implant tooth-supported fixed partial denture. J Prosthet Dent 1995 Nov;74(5):487-492.

16. Bechelli AH. The osseointegrated prosthesis-combination of osseointegrated implants and natural teeth in fixed prostheses. J Oral Implantol 1992 Feb;18(1):62-65.

17. Ozcelik TB, Ersoy AE. An investigation of tooth/implantsupported fixed prosthesis designs with two different stress analysis methods: an in vitro study. J Prosthodont 2007 Mar-Apr;16(2):107-116.

18. Rangert B, Gunne J, Sullivan DY. Mechanical aspects of a Branemark implant connected to a natural tooth: an in vitro study. Int J Oral Maxillofac Implants 1991 Summer;6(2): 177-186. 